\title{
SURVIVABILITY IN METRO RING NETWORK USING OXADM
}

\author{
Mohammad Syuhaimi Ab-Rahman* and Sahbudin Shaari
}

Photonic Technology Laboratory, Institute of Microengineering and Nanoelectronics, Universiti Kebangsaan Malaysia, 43600 UKM Bangi, Selangor, Malaysia

Received 23 March 2006

\begin{abstract}
The optical cross add and drop multiplexing (OXADM) node described in this paper increases the capacity, flexibility and reliability of the trunk-line optical network used for data communication. It features two different bandwidths which operate in working and protection line in a ring configuration. The OXADM has the same features of the conventional OADM node but with more efficiency and reliability. The OXADM prototype system focuses on providing survivability through restoration against failures, such as cable/fiber cut in optical layer with ring topology. Two types of restoration scheme have been proposed to ensure data flow continuously by means of linear/ multiplex protection and ring protection. Comparison between OXADM and OADM restoration schemes is also described. The BER characteristics were measured at $2.5 \mathrm{Gbps}$ (OC-48) and small degradation was observed, as confirmed by a comparison of these simulation results with those obtained from systems without restoration element. Restoration schemes in OXADM node are more reliable and efficient compare to conventional device due to internally routing mechanisms and no 'drop and re-add function' is needed to route the signal to alternative path or processed the signals in both electronic and optical domain as practice in conventional nodes today.
\end{abstract}

Index Term - OXADM, Metro Ring, Survivability.

\section{INTRODUCTION}

The explosive growth of data, particularly internet traffic, has led to a dramatic increase in demand for transmission bandwidth imposing an immediate requirement for broadband transport networks. Currently telecommunication networks widely employ wavelength division multiplexing (WDM) in single-mode optical fiber to interconnect discrete network locations and offer high capacity, high speed and long reach transmission capabilities $[1,6]$. Normally in wavelength routed networks, switching is performed through optical add/drop multiplexer (OADM) and optical cross connect (OXC) nodes. These nodes provide provisioning capabilities as well as protection and restoration at the optical layer $[1,3,6]$. This can be implemented by using two fibers connection; the first fiber in the working line and the second fiber in the protection line [4]. The protection line is normally without data and will be activated when fault occurs in the working line by means of dedicated protection by diverting the signal to the protection line [8]. But with the introduction of the optical cross add and drop multiplexer (OXADM) has increase the transmission capacity through the transmission using both working

\footnotetext{
* Corresponding author e-mail: rahul_devraj@hotmail.com
} 
and protection path with two different operating bandwidths. OXADM protection mechanism is more efficient compare to other restoration nodes because it does not need to 'drop and re-add' the signal to activate the redundancy. Two restoration schemes have been approached to overcome the huge data losses cause by failures due to the large transport capacity achieved by the network.

\section{RING RESTORATION SCHEME}

Providing recovery scheme at the optical layer becomes inherently attractive as the network throughput increases. Reconfigurable nodes support recovery actions as protection switching in the optical domain. The first step towards a survivable optical layer has been the use of WDM rings. These rings can be divided into unidirectional and bidirectional signal flows, the former using dedicated protection and the latter using shared protection [4]. Linear and multiplex protection schemes are designated for dedicated protection while ring protection is designated for shared protection.

In path switching, restoration of traffic is handled by the source node and the destination node. A Unidirectional Path Switched Ring (UPSR) that uses dedicated protection is referred to as a Dedicated Protection Ring (DPRing). A two-fiber DPRing uses one fiber as the working fiber and the other fiber as the protection fiber $[6,8]$. To increase the capacity of transmission, the protection fiber can also be used as working line that is employed in OXADM ring network [5].

A metro ring that utilizes shared protection is referred to as Shared Protection Ring (SPRing). A protection switching occurs on a per wavelength basis in the so-called Optical Channel Shared Protection (OCh-SPRing) or on a multiplex section level as Optical Multiplex Section Shared Protection Ring (OMS-SPRing). In an OCh-SPRing, path switching is used (restoration of traffic is handled by the source node and the destination node), and the ring is referred to as a Bidirectional Path Switched Ring (BPSR). In an OMS-SPRing, line switching is used (restoration of traffic is handled by the nodes at the ends of the failed link), and the ring is referred to as a Bidirectional Line Switched Ring (BLSR) [4, 8]. OMS-SPRing protection can be also called as ' $U$ Turn' protection scheme and will be discussed in this paper as a survivability feature comparing between OADM ring and OXADM ring network configuration.

\subsection{Conventional ring network structure}

Metro optical communication networks are built as transmission fiber lines that connect nodes, and forming rings. Traffic in metro rings typically travels over a pair of fibers, where one fiber transports the signals in one direction and the other carries the contra-directional traffic as shown in Fig. 1 (a). Figure 1 shows a schematic representation for 2 fiber metro WDM ring and functional diagram for an OADM node for bypass and protection mode.

When failure occurs in the working line, the signal will be diverted to the protection using either two types of restoration scheme; $1+1$ and 1:1 dedicated protection (Fig. 1b). In 1+1 dedicated protection, a variable splitter and a selector switch are used while in 1:1 dedicated protection, switches are used on both the transmitter and the receiver side. The signal in working path will be dropped and then re-added to protection path in the same direction with the previous path [3, 4].

For an OMS-SPRing (BLSR), in the event of a failure condition, the OADMs adjacent to the failure loop back the affected light paths on the protection channels of the ring (Fig. 1c). It is important to note that bidirectional has to use different sets of wavelengths in order to avoid wavelength conversion. In the OADM node, $\lambda_{1}$ and $\lambda_{2}$ each designate either a single wavelength or multiple different wavelengths. Here the signal from the working line will be dropped and then re-added to protection against the direction of previous path. The protection bandwidth is used to enable the wavelength in line 1 propagate in a line 2 and vice versa [4]. 

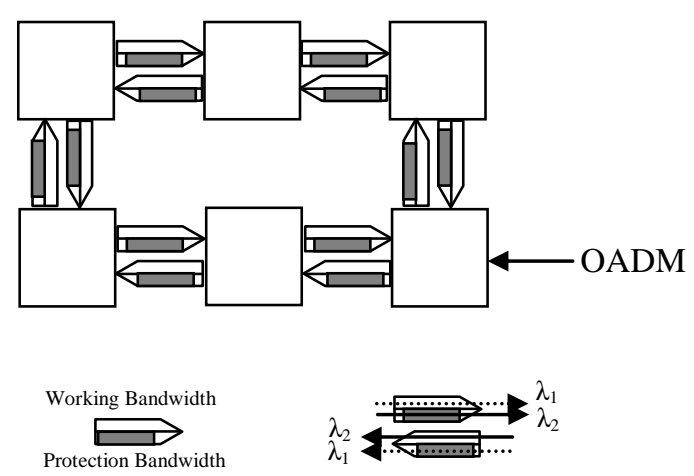

(a)

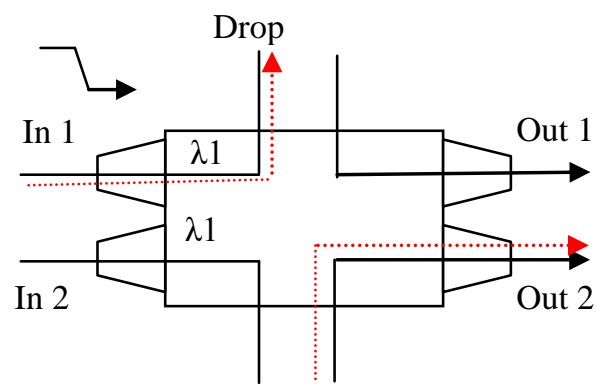

Add

UPSR: Linear protection $(1: 1 @ 1+1)$

(b)

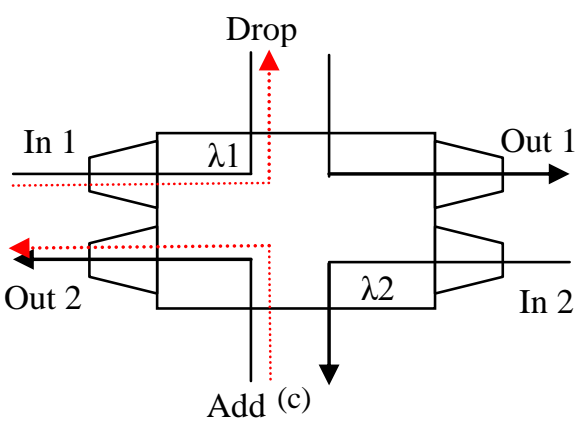

BLSR: Ring protecrion

$\begin{array}{ll}\longrightarrow & \text { Bypass mode } \\ \cdots & \text { Protection mode }\end{array}$

Fig. 1: A conventional 2-fiber metro ring (a). OADM survivability scheme - linear protection mechanism (b) ring protection mechanism (c).

\subsection{OXADM ring network structure}

In the OXADM metro ring network, the passive protection line functional is eliminated and all paths are accommodated with working wavelengths (both lines are designated with the different wavelength) (Figure 2a). The capacity is increased twice compare to the conventional metro ring network. If one of the working lines breakdown, the signal will be multiplexed with the channels from adjacent line. (Figure 2b) At the destination node, the multiplexed signal will be split to their original path. Beside that, the OXADM is also applicable for the OCh-DPRing one working line usage as shown in Figure 2 b.

When the OMS-SPRing protection scheme is activated, the signals are loop back to the second line against the direction of previous line. The switches are used to divert the wavelength to the output related path. No 'drop and re-add' operation is needed (Figure 2d). This mechanism will re-close the loop back and ensuring the signal flow continuously due to major breakdown occurs in the network. 


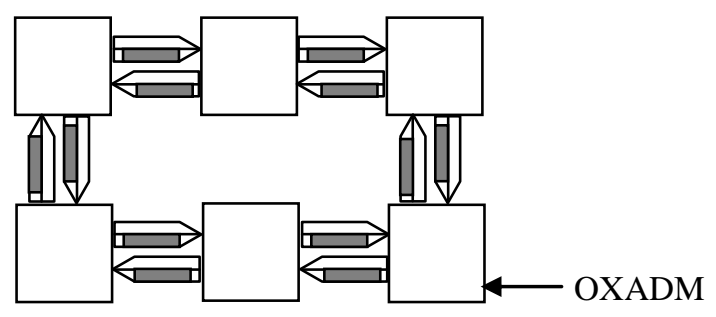

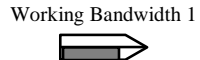

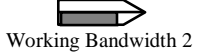

(a)
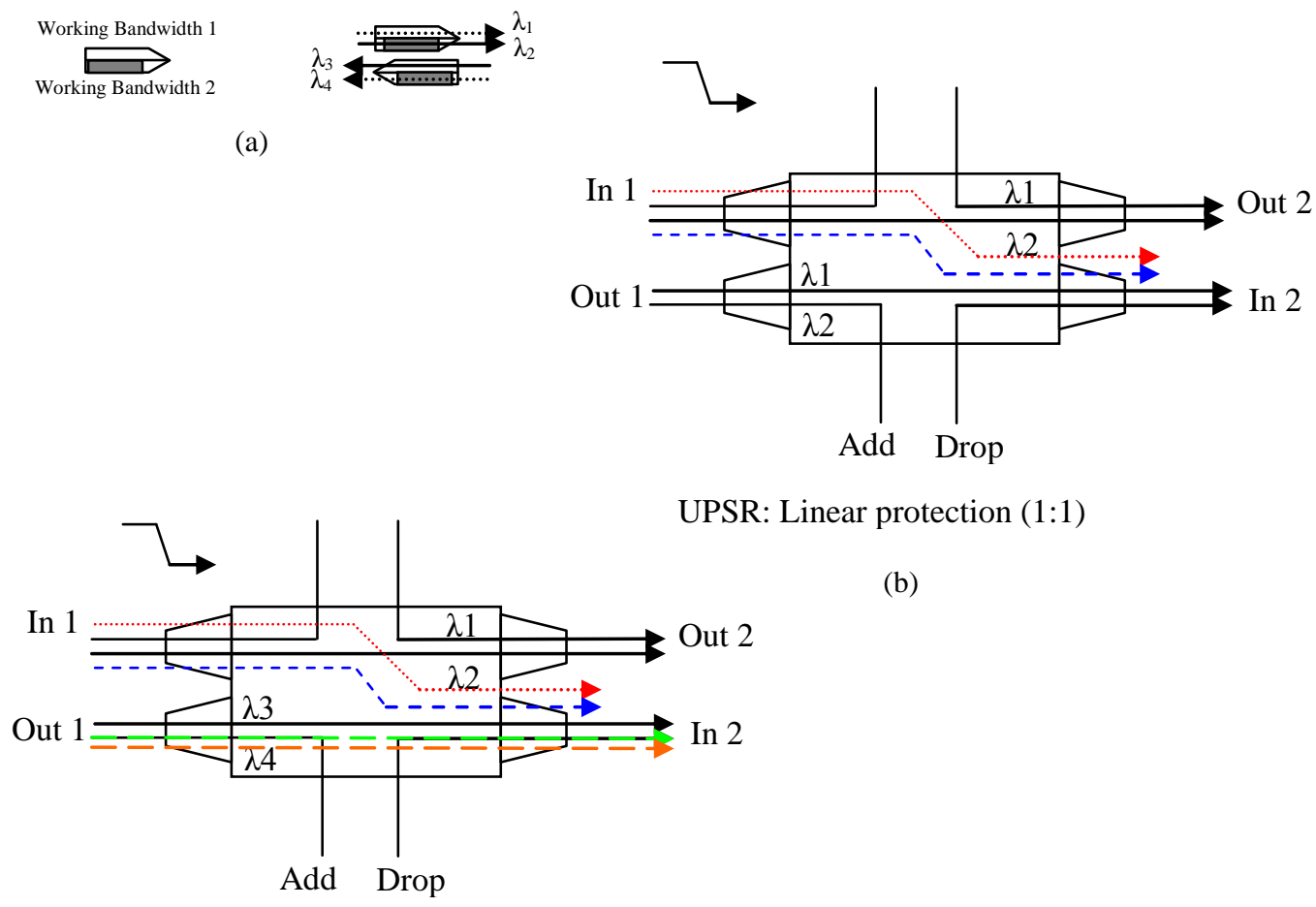

(b)

UPSR: Multiplex protection

(c)

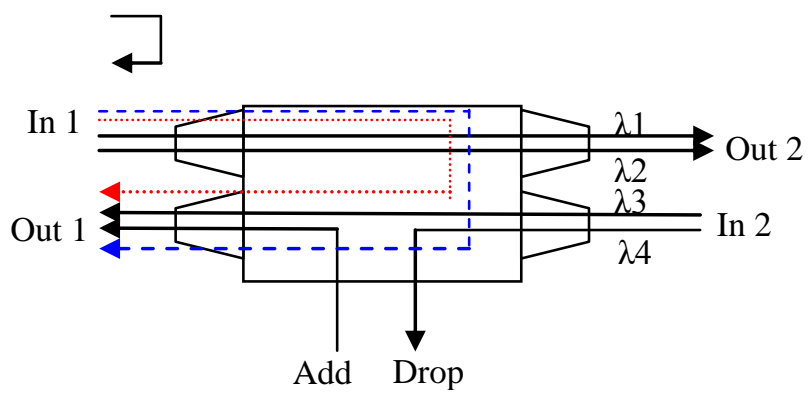

BLSR: Ring protection

(d)

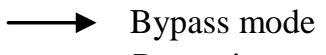

Protection mode

Fig. 2: A OXADM 2-fiber metro ring (a). OXADM survivability scheme - linear protection mechanism (b) multiplex protection mechanism (c) ring protection mechanism $(d)$. 


\section{OXADM SURVIVABILITY SCHEME}

Providing recovery scheme at the optical layer become inherently attractive as the network throughput increases. OXADM support recovery actions as protection switching in the optical domain. The first step towards a survivable optical layer has been the use of WDM rings. These rings can be divided into unidirectional and bidirectional signal flow, the former using dedicated protection and the latter using shared protection, as described below (4).

\subsection{Linear and multiplex protection}

OXADM provides a linear protection scheme to implement the dedicated protection in ring network. In path switching, restoration of traffic is handled by the source node and the destination node. Dedicated protection normally activates when one of transmission line breakdown. When a link failure occurs within the ring, the signal will be switch to the alternative route as illustrated in Figure 3 below [2][4][5]. The restoration is significant for ensuring signal flows continuously. The device is compatible with types of link usage 1:1 and $1+1$ [4]. The accumulation feature will support the shared protection to be performed in case of two different set of wavelengths going to east and west links. The restoration in $1+1$ can be called as multiplex protection.

Figure 3 depicted the activation of dedication protection when failure occurs between Node A and Node B. The affected node will switch the signals to protection route. The switching performed within the optical layer will be able to achieve high speed restoration against the failure/degradation of cables, fibers, and optical amplifiers. It is important to avoid huge losses of data and great influence upon a large number of users over a wide area.

Figure 4 and 5 depicted the output power and BER performance at 10 nodes with $70 \mathrm{~km}$ span for bypass and dedicated protection condition. Few changes in output power and BER performance was observed which shows no degradation in the BER, as confirmed by a comparison of these two simulation results bypass and linear protection state. The degradation of output power after node 5 is observed due to the not compensating between the losses and amplifiers gain. The gain of first pre amplifier and post amplifier are $3 \mathrm{~dB}$ and $22 \mathrm{~dB}$ respectively, then $7 \mathrm{~dB}$ and $21 \mathrm{~dB}$ values are applied after node 5 . This will lead to the degradation of the received power at every node.

\subsection{Ring protection}

Now suppose an entire node fails, or both the primary and the protection fibers in a given span are severed, which could happen if they are in the same cable duct between two nodes. In this case, the nodes on either side of the failed internodal span internally switch the primary-path connections from their receivers and transmitter to the protection fiber, in order to loop traffic back to the previous node. This process again form a closed ring, but now with all of the primary and protection fibers in use around the entire ring as shown in Figure 6. The protection scheme is called shared protection [4].

The shared protection is activated when either both fiber or node breakdowns. In the event of a failure condition, the OXADM adjacent to the failure loop back the affected signal on the protection route of the ring. The ' $U$ turn' mechanism is applied in OMS-SPRing (BLSR) [4].

OXADM implements the shared protection by using internal route that is activated by $\mathrm{E}$ switches. The mechanism is different compare to other devices such as OADM that use their drop function and re-inject the signal to add port to perform ' $U$ turn' protection but the node based on OXC architecture cannot perform this mechanism. Thus it is not suitable to be applied in ring topology network. 
Figure 6 depicted the shared protection is activated at node 3 represents the survivability when failure occurs at both transmission line. Because of the simulator limitation, the failure of two fibers or node cannot be designed. But the mechanism of $U$ turn is performed by node 3 bidirectional to represent the activation of shared protection by the affected node adjacent to the breakdown area. Figure 7 and 8 show the output power and BER performance by comparing the bypass and ring protection state respectively. There are $2 \mathrm{~dB}$ different in output power between activation of ring protection and normal condition. The network has undergone performance test at 2.5 Gbps (OC-48) with BER less than $10^{-9}$. The minimum BER can be achieved by increase amplification gain. The gain for pre-amplifier and post-amplifier are $7 \mathrm{~dB}$ and $23 \mathrm{~dB}$ respectively with the first pre amplifier gain is $3 \mathrm{~dB}$ ensure the power will increase gradually by the end of the node. The increasing of output power will be slower the degradation of bit error rate.

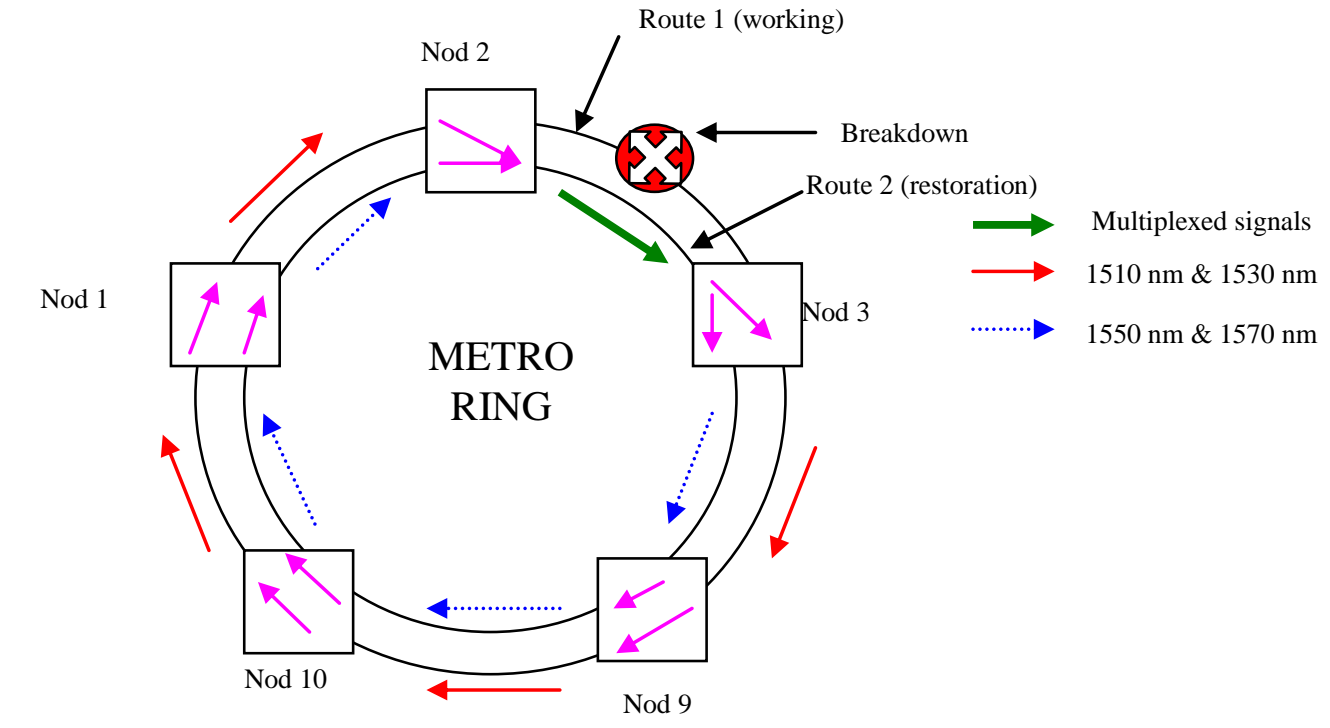

Fig. 3: Dedicated protection mechanism in metro ring network. When a link failure occurs within the ring, the affected is switched over to the protection path.

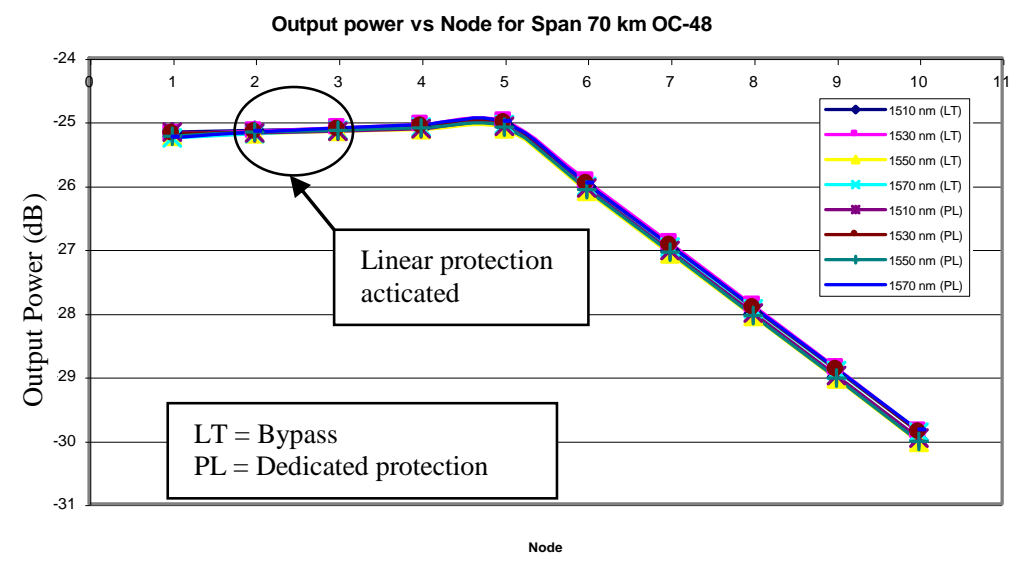

Fig. 4: The output power at 10 nodes with $70 \mathrm{~km}$ span for bypass and dedicated protection mechanisms which occurs in between node 2 and node 3. 


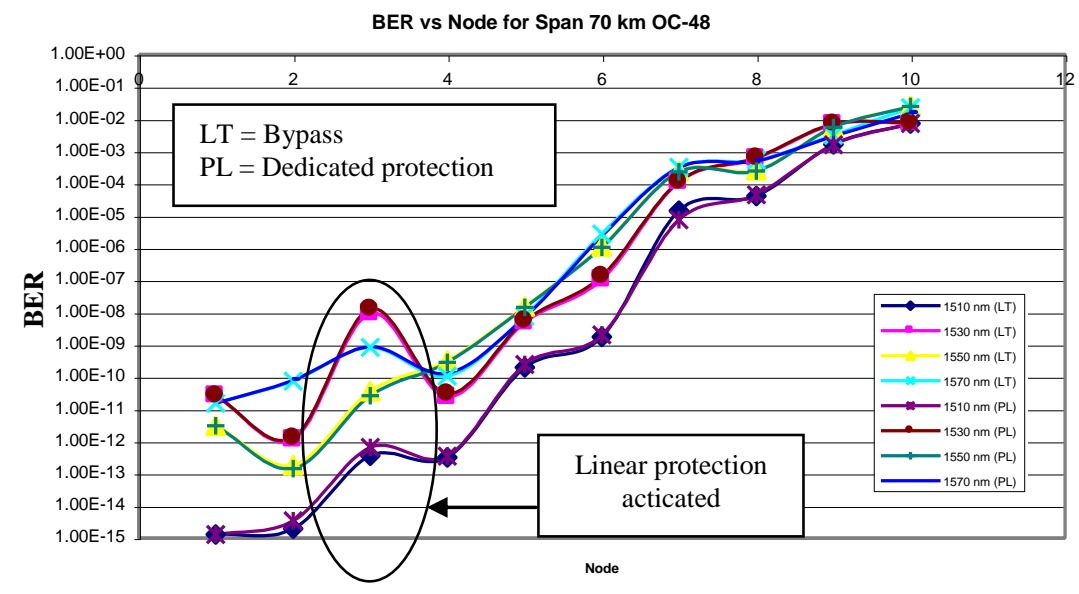

Fig. 5: The BER performance at 10 nodes with $70 \mathrm{~km}$ span for bypass and dedicated protection mechanisms which occurs in between node 2 and node 3.

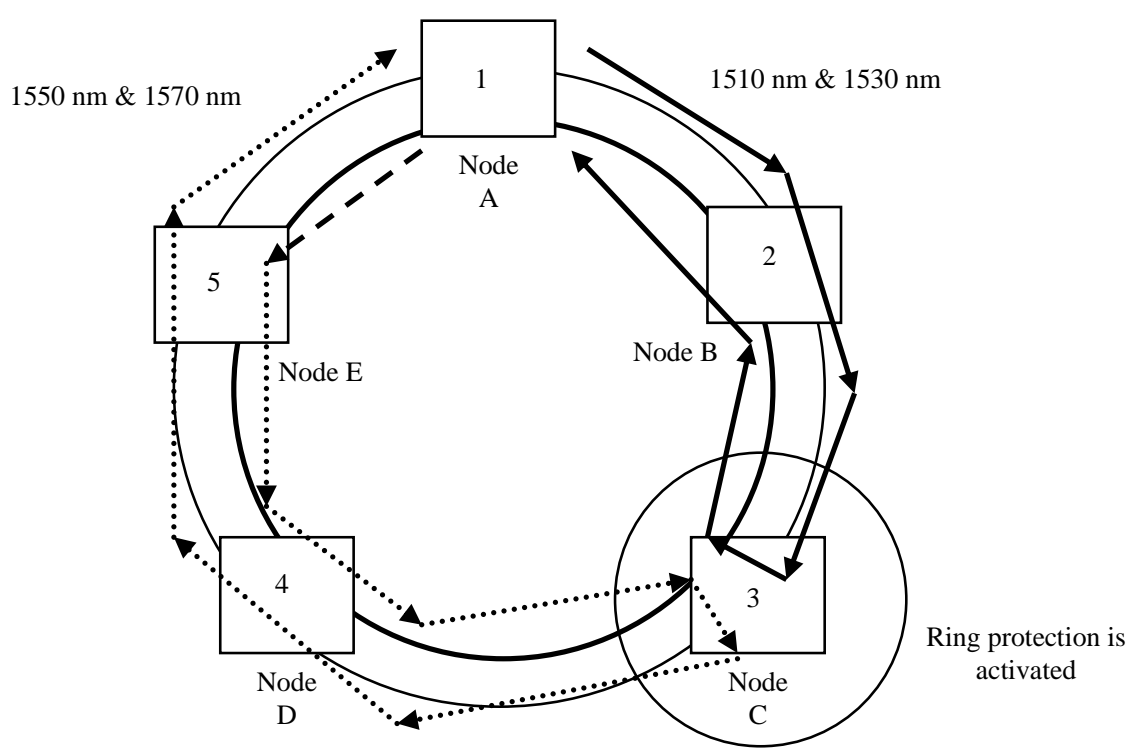

Fig. 6: The activation of shared protection to re-close the network in case of either both line or node is breakdown. The 'U-turn' mechanism is activated predominantly in optical domain. In real condition the loop closer will be done by adjacent failure nodes (In this case Node 2 and Node 4 will be activated).

\section{CONCLUSION}

This paper described our recent approach toward the advanced ring metropolitan network through the development of an optical cross add and drop multiplexer (OXADM) switch. The OXADM focuses on providing survivability through restoration against failure by means of linear/multiplex and shared protection that are activated according to the condition of failure. The BER characteristics were measured at $2.5 \mathrm{Gbps}$ (OC-48) and small degradation was 
observed, as confirmed by a comparison of these simulation results with those obtained from systems without restoration element. Restoration scheme in OXADM node is more reliable and efficient compare to conventional device due to internally routing mechanisms and no 'drop and re-add function' is needed to route the signal to alternative path when failure occurs in working path.

Though successful proposal have been obtained, it is apparent that there are still many issue which have to be solved in order to establish complete optical layer routing and supervision are also expected to be integrated in the optical layer. For the global interworking of the optical layer network, a development which satisfies the requirements of international standardization such as ITU-T of important. UKM photonics will continue to make efforts toward the development of advanced customer access network through realizing the restoration system.

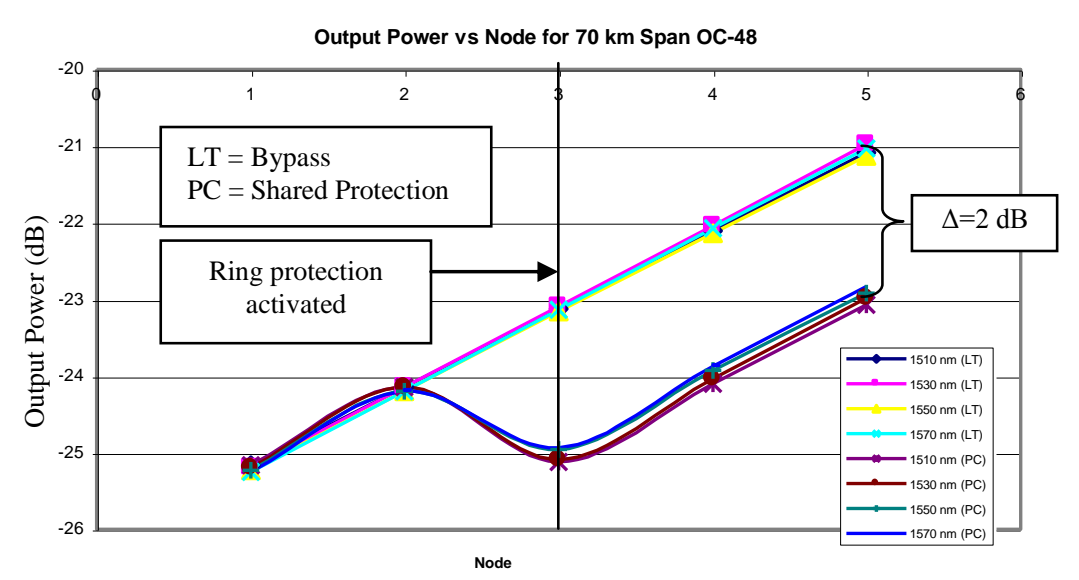

Note: Nodes configuration according to signals flow in real failure condition:

To east

Node $4=$ Node 2

Node $5=$ Node 1

To west

Node $1=$ Node 5

Node $2=$ Node 4

Fig. 7: The output power at five nodes with the activation of shared protection at node 3 (Figure 6). There are degradation about $2 \mathrm{~dB}$ as compare to the bypass state.

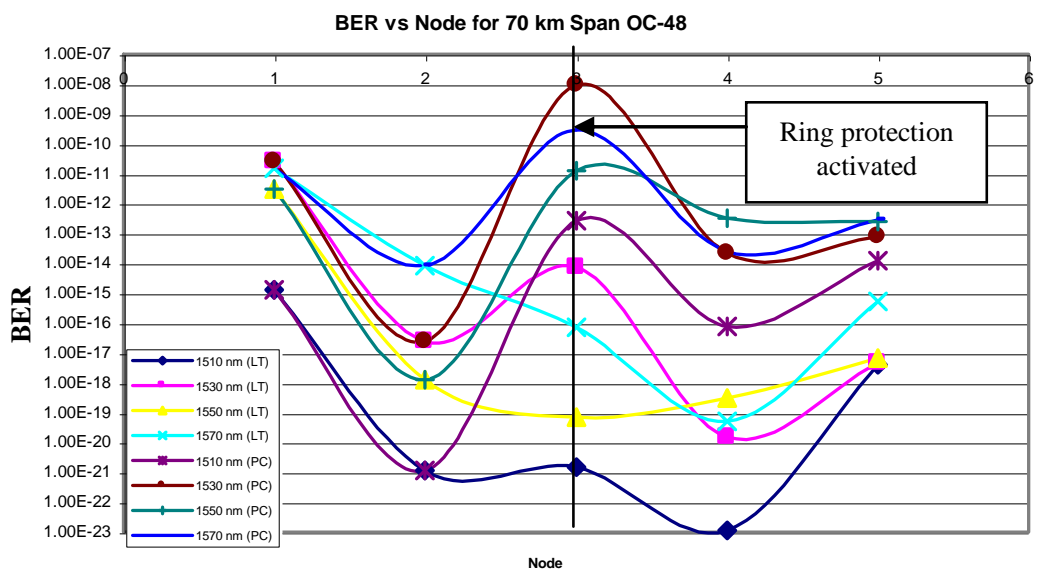

Fig. 8: The BER performance at five nodes with the activation of shared protection at node 3. 


\section{ACKNOWLEDGEMENT}

This project is supported by Ministry of Science, Technology and Environment, Malaysia Government, through the National Top-Down Project fund and NSF student fund.

\section{REFERENCES}

1. Tzanakaki, A., Zacharopoulus, I., and Tomkos, I. (2003), Optical Add/Drop Multiplexers and Optical Cross-Connects for Wavelength Routed Network. ICTON 2003, pp. 41-46.

2. Mutafungwa, E. (2000), An Improved Wavelength-Selective All Fiber Cross-Connect Node. IEEE Journal of Applied Optics. pp. 63-69.

3. Tsushima, H., Hanatani, S., Kanetake, T., Fee, J.A., and Liu, S.a. (1998), Optical crossconnect system for survivable optical layer networks, Hitachi Review, vol. 47, No. 2. pp. 85-90.

4. Eldada, L. and Nunen, J.v. (2000), Architecture and Performance Requirements of Optical Metro Ring Nodes in Implementing Optical Add/Drop and Protection Functions. 2000 Telephotonics Review.

5. Rahman, M.S.A. and Shaari, S. (2004), Modeling of Planar Lightwave Circuit OADM for CWDM, Proceeding 2004 Postgraduate Conference, vol. 1, pp. 116-120.

6. Shiragaki, T., Henmi, N., Kato, T. (1998), Optical Cross-Connect System Incorporated with Newly Developed Operation and Management System. Journal on Selected Areas in communications, vol. 16, No. 7, pp. 1179-1189.

7. Shen, Y., Lu, K., and Gu, W. (1999), Coherent and Incoherent Crosstalk in WDM Optical Networks. IEEE Journal Lightwave Technology, vol. 17, No. 5, pp. 759-764.

8. http://www.acterna.com/global/products/description/ANT/ 